\section{P5.15 PREPARING FOR PREP: ESTIMATING THE NEED FOR HIV PRE-EXPOSURE PROPHYLAXIS AMONG MEN WHO HAVE SEX WITH MEN USING SEXUAL HEALTH SURVEILLANCE DATA IN ENGLAND}

${ }^{1}$ Holly Mitchell, 'Sarika Desai, 'Hamish Mohammed, 'Koh Jun Ong, 'Martina Furegato, ${ }^{2}$ Nigel Field, ${ }^{1} 0$ Noel Gill. ${ }^{1}$ Public Health UK; ${ }^{2}$ University College London, UK

\subsection{6/sextrans-2017-053264.631}

Introduction To inform public health planning for a large-scale PrEP trial in England, we estimated the need for HIV preexposure prophylaxis (PrEP) among men who have sex with men (MSM) attending sexual health clinics.

Methods National STI surveillance data from the genitourinary medicine clinic activity dataset (GUMCADv2) were used to estimate the annual number of HIV-negative MSM who had a HIV test in the past year (which will be a criterion for accessing PrEP in England), for 2010-2015. To estimate the number and proportion of all MSM needing PrEP, we used bacterial STI diagnosis in the past year as a proxy for high-risk behaviour, and estimated HIV incidence (per 100 person-years) in both groups. We used these data to understand the likely geographical distribution of MSM who might need PrEP within the 152 English counties.

Results The number of HIV-negative MSM attending sexual health clinics increased by $68 \%$ from 69392 in 2010 to 116546 in 2015, and the number of HIV-negative MSM with a prior HIV test nearly doubled from 14643 to 29023 in the same period. Among HIV-negative MSM with a prior HIV test, the number with a recorded bacterial STI (past year) increased from $4365(30 \%)$ in 2010 to $10,276(35 \%)$ in 2015 (33\% on average). HIV incidence among MSM with a prior HIV test was 1.9 (95\% CI 1.6-2.2) per 100py compared to $3.3(2.7-4.0)$ per 100 py in MSM with a prior HIV test and history of bacterial STI. The number of MSM in need of PrEP (according to bacterial STI history) was 200 men in $4 \%(6 / 152)$ of counties.

Conclusion We estimated that the need for PrEP among MSM in England in 2015 might be around 10000 individuals with an annual HIV incidence of 3\%. Need for PrEP was highly concentrated; in most English counties, the number of MSM with a prior HIV test was small, and only $33 \%$ of these men might be clinically assessed as eligible for PrEP. These data illustrate how the population need for PrEP might be estimated in advance of a national trial, and will inform future evaluations at a population level.

\section{P5.16 COMBINATION OF INHIBITORS OF CHAPERONE ACTIVITY AND CHAPERONE EXPRESSION FOR PREVENTION OF HIV-1 REACTIVATION FROM LATENCY}

A Kabakov, E Zotova, A Sobenin. Biomedical Research Centre Bion, Russia

\section{$10.1136 /$ sextrans-2017-053264.632}

Introduction In vivo, the state of latency allows HIV-1 to persist in cellular reservoirs and avoid eradication. Intracellular heat shock protein 90 (Hsp90) was shown to contribute to HIV-1 reactivation from latency, so that cell-permeable inhibitors of the Hsp90 chaperone activity can prevent this reactivation and be considered as potential anti-AIDS agents. However, the Hsp90 activity inhibitors provoke up-regulation of inducible Hsp90, Hsp70, Hsp27 and we suggested that such accumulation of chaperones in cellular reservoirs assists the virus and impairs the beneficial effects of Hsp90-inhibiting treatment. Here we examined whether the suppressive action of Hsp90 inhibitors on the HIV-1 reactivation is enhanced by targeting the Hsp induction and/or the chaperone function of Hsp70.

Methods The HIV-1 reactivation was studied in cultured J-Lat cells. 17AAG and AUY922 were used as the Hsp90 activity inhibitors. The Hsp accumulation in the Hsp90 inhibitortreated cells was blocked by co-treatments with quercetin or KNK437. The Hsp70 chaperone function was inhibited by 2 phenylethynesulfonamide (PES).

Results Inhibition of the Hsp90 chaperone activity with 17AAG or AUY922 does suppress the HIV-1 reactivation in the drug-treated cells but this is also accompanied by the upregulation of Hsp90, Hsp70 and Hsp27. In the case of inhibitory co-treatments (17AAG or AUY922 + quercetin or KNK437 + PES), no increase in the cellular Hsp levels occurred despite of the dysfunction of Hsp90-,Hsp70-dependent chaperone machine. Such a combination of the inhibitors simultaneously targeting the chaperone activities of Hsp90 and Hsp70 and the Hsp induction much stronger suppressed the chaperone-dependent HIV-1 reactivation, as compared with the action of Hsp90 inhibitors alone.

Conclusion Intracellular Hsp70 appears to contribute to the HIV-1 reactivation from latency. The suppressive effects of Hsp90-inhibiting drugs on the HIV-1 reactivation from latency can be enhanced by parallel inhibiting both the Hsp induction and the Hsp70 chaperone activity.

\section{P5.17 ATTITUDES OF CHURCH LEADERS ON HIV PREVENTION AMONG THE PRESBYTERIAN CHURCH LEADERS OF AIZAWL, MIZORAM, INDIA}

Lalchhanhima Ralte. Presbyterian Church of Mizoram, India

\section{$10.1136 /$ sextrans-2017-053264.633}

Introduction Knowledge about Church leader's attitudes towards HIV prevention is essential to understand the factors that enable them to take on leadership role in facilitating HIV prevention efforts. Church leaders can have a significant contribution in a Christian dominated state like Mizoram. The study aims to explore attitudes of church leaders on HIV prevention among the Presbyterian Church leaders in Aizawl City, Mizoram.

Methods A Cross sectional study using in-depth interviews and focus group discussions were used. From 15 Presbyterian churches randomly selected all over Aizawl city, 293 Church leaders representing the four groups of leadership (Pastor/ Elder, Women, Youth and Men) completed a self administered questionnaire along with 12 in-depth interviews and 3 focus group discussions. Bivariate analysis was done to identify associated factors.

Results The proportion of Church leaders willing to advocate condom use for HIV prevention was 34.0 percent. Around 97.3 percent agreed that Church leaders should be concerned and intervene in HIV prevention. Nearly 90.4 percent felt it should be discussed in Church services. About 70 percent of the Church leaders, Biblical disobedience leads to HIV infection and almost 80 percent felt homosexuals deserve HIV infection. Abstinence (77.1\%), marital fidelity (22.2\%) and condom use $(0.3 \%)$ were the preferred choice for HIV prevention. Although 66.9 percent agreed with the Church 
statement that condoms encourage pre-marital sex, more than 80 percent agreed that condom prevents spread of HIV infection. Personal contact with PLHIV does not seem to have positive influence with willingness to advocate condom use.

Conclusion Church leaders agreed that they have responsibilities towards HIV prevention but were restricted by the Church doctrine. Disobedience to Biblical teachings was believed to result in HIV infection which is seen as something sinful. Misconceptions around HIV persist irrespective of awareness programs within the Church. Sex education from early childhood, using Church media and educating Church leaders were recommended for effective HIV prevention.

\section{P5.18 A GLOBAL ESTIMATE OF THE ACCEPTABILITY OF PRE- EXPOSURE PROPHYLAXIS FOR HIV AMONG MEN WHO HAVE SEX WITH MEN: A SYSTEMATIC REVIEW AND META-ANALYSIS}

'Lei Zhang, ${ }^{2}$ Peng Peng. ${ }^{1}$ Melbourne Sexual Health Centre, Australia; ${ }^{2}$ School of Public Health, Monash University, Melbourne, Australia

\subsection{6/sextrans-2017-053264.634}

Introduction Pre-exposure prophylaxis (PrEP) is a new biomedical intervention for HIV prevention. This study systematically reviews the acceptability of PrEP among men who have sex with men (MSM) worldwide.

Methods We searched PubMed database to identify Englishlanguage articles published between July 2007 and July 2016, which reported the acceptability of PrEP and associated population characteristics. Meta-analysis was conducted to estimate a pooled rate of acceptability, and meta-regression and subgroup analysis were used to analyse heterogeneities.

Results Sixty-eight articles were included. The estimated acceptance of PrEP was 58.7\% (95\% confidence internal (CI): $53.4 \%-63.8 \%$ ) among MSM worldwide and showed no significant difference between developed and developing countries. MSM who were younger ( $4 / 5$ studies, range of adjusted odds ratio $(\mathrm{aOR})=1.49-3.47)$, better educated $(7 / 7$ studies, $\mathrm{aOR}=1.49-7.70)$, wealthier (3/3 studies, $\mathrm{aOR}=1.31-13.03)$, previously aware of $\operatorname{PrEP}(4 / 4$ studies, aOR $=1.33-3.30)$ and had greater self-perceived risk of HIV infection (4/5 studies, $\mathrm{aOR}=1.20-4.67)$ showed significantly higher acceptance of PrEP. Male sex workers (84.0\% [26.3-98.7\%]\%] were more likely to accept PrEP than general MSM. Self-perceived low efficacy and concern about side effects, adherence, affordability, and stigma from health providers and society were main barriers for accepting PrEP.

Conclusion This review identifies a moderate acceptability of PrEP in MSM both developed and developing country settings. Efficacy, individuals' perception of HIV risk and level of experienced stigma determine its acceptance.

\section{P5.19 HEALTH NEEDS OF WOMEN WHO HAVE SEX WITH WOMEN AND ACCESS TO HEALTH SERVICES}

Marli Teresinha Cassamassimo Duarte, Ana Paula Freneda de Freitas, Juliane Andrade, Mariana Alice De Oliveira Ignacio. Universidade Estadual Paulista, Brazil

\subsection{6/sextrans-2017-053264.635}

Introduction Access and host are essential in order to satisfactorily intervene in the population health and the Brazilian literature has few studies approaching the access to services and sexual and reproductive care among women who have sex with women (WSW). The aim of this study was to analyse the access to health services and sexual and reproductive health care of WSW in public health policies.

Methods Cross-sectional, descriptive and analytical study. The data were obtained by interview, gynaecological and blood laboratory examination for sexually transmitted infections (STI) diagnosis, from January 2015 to December 2016.The descriptive statistics and the quantitative method of Bardin were used to analyse the access to health services. Sexual and reproductive health care was analysed by linear and multiple regression models.

Results The intentional sample was 149 WSW and the median age was 27 years-old (18-62). There was a predominance of white women (74.5\%), non-union (73.2\%), who had paid activity (73.2\%) and eight years or more of completed study $(96,0 \%)$. The majority $(84.0 \%)$ used the public health service, $22.6 \%$ sought preventive actions. The difficulties encountered in the health services care were reported by 77 women, resulted in 118 responses with $28.8 \%$ associated with difficulty by long waiting time and $7.6 \%$ by lack of preparation of the professional/service for MSM.Regarding sexual and reproductive care, $71.8 \%$ had sexual intercourse after consumption of alcohol and/or illicit drugs, $12.1 \%$ did not use a condom and $51.7 \%$ had at least one STI. Age was a factor associated with the care score $(p=0.001, \beta=1.36 \mathrm{CI}=0.57$ 2.15) and no differences were observed in the MSM health care score with a history of sexual intercourse with men compared to those who only had women.

Conclusion Beside the difficulties of access to health services already experienced by women in general, there is a high programmatic in addition to individual and social vulnerability. This study indicates the need for effective strategies for the proposed policies for WSW.

\section{P5.20 HPV VACCINATION INTENTION AMONG FEMALE SEX WORKERS IN AMSTERDAM, THE NETHERLANDS}

${ }^{1}$ MF Schim Van Der Loeff, E Marra, ${ }^{1} \mathrm{~L}$ Van Dam, ${ }^{1} \mathrm{~N}$ Kroone, ${ }^{1} \mathrm{M}$ Craanen, ${ }^{1} \mathrm{~A}$ Van Dijk, ${ }^{1} \mathrm{CJ}$ Alberts, ${ }^{2} \mathrm{GD}$ Zimet, ${ }^{3} \mathrm{TGWM}$ Paulussen, ${ }^{1} \mathrm{~T}$ Heijman, ${ }^{1} \mathrm{~A}$ Hogewoning, ${ }^{1} \mathrm{GJB}$ Sonder, ${ }^{1} \mathrm{HJC}$ De Vries. ${ }^{1}$ GGD Amsterdam, The Neederlands; Indiana University, USA; ${ }^{2} T N O$

\subsection{6/sextrans-2017-053264.636}

Introduction Female sex workers (FSW) are at risk for HPVinduced diseases but are currently not targeted by the HPV vaccination program in the Netherlands. We explored the determinants of their intention to get vaccinated against HPV in case vaccination would be offered to them in the near future.

Methods In 2016, FSW aged >18 years having an STI consultation with the Prostitution and Health Centre (P and G292) in Amsterdam, either at the clinic or at their working location, were invited to complete a questionnaire assessing the socio-psychological determinants of their HPV vaccination intention (scale ranging from -3 to +3 ). Determinants of HPV vaccination intention were assessed with uni- and multivariable linear regression. Additionally, we explored the effect of out-of-pocket payment on intention.

Results Between May and September 2016, 293 FSW participated; 98 (34\%) worked in clubs/private houses/massage salons, $111(38 \%)$ worked at 'prostitution windows', and 81 $(28 \%)$ worked as escorts or from home. The median age was 